Alkistis Elliott-Graves

University of Helsinki (TINT)

alkistis.elliott-graves@helsinki.fi

\title{
Generality and Causal Interdependence in Ecology
}

\author{
Penultimate version - Forthcoming in Philosophy of Science
}

\begin{abstract}
A hallmark of ecological research is dealing with complexity in the systems under investigation. One strategy is to diminish this complexity by constructing models and theories that are general.

Alternatively, ecologists can constrain the scope of their generalisations to particular phenomena or types of systems. However, research employing the second strategy is often met with scathing criticism. I offer a theoretical argument in support of moderate generalisations in ecological research, based on the notions of interdependence and causal heterogeneity and their effect on the tradeoff between generality and realism.
\end{abstract}

Acknowledgements: I would like to thank Roberta Millstein, Steve Peck, Chris Eliot, Adrian Currie, an anonymous reviewer and the audience at the 2016 PSA for helpful and constructive comments. 


\section{Introduction}

The ability to produce results and theories that apply generally is a central goal of science. A popular view in sciences that study complex systems is that achieving generality reduces system complexity, making systems more intelligible and easier to model, thereby increasing our ability to construct successful explanations and predictions. In ecology, the pursuit of generality has a history that is almost as long as the history of the discipline itself. For some, ecology only became a truly scientific discipline when it applied simple, general models from physics to ecological populations (Kingsland 1995). Before these general models were incorporated into the field, ecology was simply a collection of observations of nature (Shrader-Frechette and McCoy 1993). Consequently, the more ecology could be made to resemble physics, the higher its scientific worth.

However, ecologists often find it difficult or even impossible to achieve the kinds of generalizations common in other fields of science. This has led to widespread criticism of the field as unscientific, especially sub-disciplines such as community ecology, ecosystem ecology and invasion biology (Peters 1991; May 2004). One response to the criticism is an ongoing quest for generality. If we discount the rhetoric ${ }^{1}$, the overall trend of this quest seems to be moving towards more constrained or modest levels of generality. The aim of this paper is to provide philosophical support of this trend. While I do not deny that there is a place for

\footnotetext{
${ }^{1}$ Often, the claim to generality in the introduction of a paper is much more grandiose than the actual scope of the generalization being proposed in the main text (see Borer et al. 2014, 65).
} 
extensive generalizations in ecological research, it is not the only important goal. Achieving generality often comes at a cost; a sacrifice of realism and precision that decrease the explanatory and predictive power of ecological models. Therefore, some proportion of ecological research should be devoted to the pursuit of the other goals, even if this comes at the expense of generality.

I start with an overview of the most important milestones in the quest for ecological generality, highlighting the trend towards modest generality (section 2). I then examine the reasons for which generality is disproportionally difficult to achieve in ecology (section 3). Following Levins (1969), I argue that complexity creates tradeoffs between generality, realism and precision, yet the tradeoff is significantly magnified by the additional factors of interdependencies and causal heterogeneity. In light of these characteristics of ecological systems, I argue that modest generalizations are valuable qua generality for ecological research (section 4).

\section{The Quest for Ecological Generality}

The debate around the existence and nature of ecological laws became the first arena for discussions of ecological generality. The main opposition to the quest for general ecological laws stemmed from the observation that ecological systems are complex, spatially and temporally variable, and influenced by non-deterministic factors (Railsback and Grimm 2011; Cooper 1998). Proponents of this view argued that the search for laws was futile and that 
ecologists should focus instead on gaining in-depth knowledge about particular systems (Lawton 1999). For some, this was a worthwhile and valuable pursuit (Travis et al 2014), yet for others, it necessitated a downgrading of ecology (or its subdisciplines) from the status of a true science (Valéry et al. 2013).

For the optimists, the problem was not in ecology, but in the particular conception of laws. This view is supported by refinements in the philosophical conception of laws of nature, where laws need not be universal, but can accommodate pragmatic constraints or exceptions (Mitchell 1997). This line of the debate continues to the present, with increasingly refined (but arguably more relevant and useful) conceptualizations of "general laws" (Colyvan and Ginzburg 2003; Linquist et al. 2016).

For the purposes of this paper, I will focus on the other line of the debate, which shifted the focus away from the definition of laws and towards achieving generality by other means. In an interesting mirroring of philosophical accounts of explanation, the quest for generality in ecology moved from laws towards general theories that could unify a number of disparate phenomena. Many such theories (aka general frameworks) have been proposed, including keystone species, allometry and the metabolic theory. They are meant to identify patterns in nature that encompass and thus explain many diverse and particular phenomena, just as Darwinian evolution explains the existence of a variety of traits as stemming from common set of underlying mechanisms (Kitcher 1981). 
Another strategy is the search for general models (that apply to many systems) (Levins 1969; Matthewson 2011). Sometimes this strategy goes hand in hand with the search for a unifying theory, i.e. the general theory is applied to actual situations through a corresponding model or closely related group of models. In these cases, the models are often ones incorporated from physics (e.g. Lotka-Volterra models).

The interesting point for this discussion is the way in which these models achieve generality. They are often simple, containing few parameters that correspond to parts or properties of the real world (Evans et al. 2013; May 2004). The rationale is that there are fundamental underlying factors or dynamics common to many diverse ecological phenomena, whereas differences between individual cases come from confounding details or 'idiosyncrasies' of particular systems. Therefore, the purported advantage of uncovering these fundamental factors is two-fold. It allows scientists to distinguish between 'real' causal factors and mere details, but it also provides a reasonable way to unify the different phenomena under one general model.

This position is quite common in ecology, though not ubiquitous. Another method for seeking generality through modelling is to look for patterns that generalize across systems. For example, pattern-oriented Individual Based Models start from observations of patterns in real-world systems, build models based on those patterns and examine the extent to which these patterns appear in other systems (Grimm et al. 2005). These are often 'bottom-up' approaches whose generalizations are not as extensive as the 'top-down' approaches 
mentioned above (Cooper 1998). Importantly, advocates of this approach view the limited generalizability of their models as a feature rather than a bug, as they believe it ensures that their models exhibit optimal levels of complexity.

Experimental ecologists, who seek to generalize the results of their experiments, employ a similar method. They start from particular, finely specified experimental results and attempt to generalize 'upwards' from them, with the hope of finding patterns across different systems. As before, the level of generality expected from this approach is usually quite constrained, as its scope is limited to variation within particular types of phenomena, such as disturbance (Peters et al. 2006), plant-soil feedback (Casper and Castelli 2007) and migration (Kelly and Horton 2016). Occasionally, some ecologists seek to generalise further, by examining whether these patterns can be incorporated into pre-existing unifying theories (e.g. Thrush et al. 2000; Borer et al. 2014), though it is more common to focus on integrating data and methods so as to achieve a more modest level of generality (Richardson and Rejmánek 2004; Phillips et al. 2016).

This discussion is not meant to provide an exhaustive list of strategies for achieving generality in ecology, but a representative sample of the most important ones. It also demonstrates the trend towards decreasing the expected scope of a generalization. The pursuit of laws of nature gave way to more restricted law-like statements that admit exceptions, or to theories that unify some parts or aspects of ecological interactions. As the generality of many 
of these theories is being contested, research has shifted towards bottom-up approaches that aim to encompass narrower classes of phenomena or systems.

This shift reveals an important point about the status of generality in the field of ecology. Despite the theoretical and practical difficulties associated with the pursuit of generality, a significant number of ecologists continue to strive for it in their research, by revising the very notion of the term generality to make it compatible with ecological reality. Whether or not this is merely a reaction to the pessimists who use the lack of extensive generality as a criticism of the field, it reveals just how much ecologists value the generality. I will pick up this issue again in section 4, after a detailed examination of the difficulties associated with achieving generality in ecological research.

\section{Complexity, Heterogeneity and Interdependence}

The most commonly cited culprit for difficulties in ecological research, especially with respect to generality and predictability, is complexity (see Beckage et al. 2011; Odenbaugh 2003; Mikkelson 2001). Ecological systems are made up of many interacting parts but we cannot capture all of the parts, properties and interactions between them in our experiments, nor represent all of them in our models. Perhaps the most compelling account of complexity and its effect on generality is Levins's view of tradeoffs in modelling (Levins 1969, 1993).

According to Levins, there are three desiderata that modellers aim to maximize in their models: generality is understood as the applicability of a model to many systems in the world. 
A model is precise when its results and predictions are finely specified. Completely realistic models accurately capture the causal structure of the world, i.e. are not overly simplified or idealized. ${ }^{2}$ The problem is that these three desiderata trade off against each other so that modellers can only maximize two out of the three. This gives rise to three distinct strategies for modelling, and three corresponding types of models. For the purposes of brevity, I will not outline all three strategies, but focus on the two that demonstrate the underlying reasons for the difficulties in achieving generality.

Type II models (e.g. fisheries models, or ecosystem network models) maximize precision and realism at the expense of generality (Levins 1993, 1966). They are often constructed with a particular system in mind and contain many factors present in the real-world target system. For example, a three-tiered trophic chain (plant, herbivore, predator) has a "direct positive link" between plant and predator, as the abundance of the plant serves as a signal to attract the predator (Levins 1993, 68). A model that maximizes realism will include this link, with the aim of maximizing the explanatory and/or predictive power of the model. However, this will

\footnotetext{
${ }^{2}$ I should note that Levins did not provide definitions of these terms. These are reconstructions, from the 1966 and 1993 papers, compatible with other reconstructions in the literature (e.g. Odenbaugh 2003). For example, Levins states that realism can be increased by adding independent variables, dependent variables, new links between variables, by relaxing simplifying assumptions, or by restricting the domain of application of a model $(1993,548$ 549). Type II models are highly unrealistic because "they omit time-lags, physiological states, and the effect of a species' population density on its own rate of increase", and they contain assumptions analogous to "frictionless systems or perfect gasses" $(1966,422)$. In these models, "factors we know to be operating are ignored, circumstances such as symmetry that are uncommon in reality are assumed" $(1993,552)$.
} 
make the model less applicable to systems that do not have this link, thus reducing the model's generality.

On the other hand, Type I models (perhaps the most common in population ecology) achieve generality by omitting all the causal factors specific to particular systems and including only those that are common across systems. This provides the added benefit of distinguishing between mere causal details (idiosyncrasies) and the core causal factors that give rise to the phenomenon. For example, the logistic equation of population growth shows how populations grow when they are limited by the carrying capacity of the environment. This dynamic is thought to be the core factor of population growth, present in all populations, even when other factors are also present. Thus, the model that describes the process common to all populations is general.

The tradeoff account provides the basic insight of how complexity causes difficulties for generalizing in ecology, but does not adequately capture the extent of these difficulties. First, many ecological models incorporate a great extent of complexity (type II models). Second, scientists in other fields that study complex systems, such as physics and chemistry, do not seem to face such extensive tradeoffs (Matthewson 2011). In fact, if we look more closely at the language in ecologists' complaints concerning the pursuit of generality, we will notice that ecological systems and the factors that operate within them are characterised as 'idiosyncratic' (Lawton 1999; Beckage et al. 2011). That is, the factors that affect ecological systems are not 
just intertwined with many others, but they or their effects are sometimes peculiar and uncommon.

Matthewson (2011) captures one aspect of the idiosyncratic nature of ecological systems. For Matthewson, ecological systems are not merely made up of many interacting parts (complexity), but the parts themselves vary across systems (heterogeneity). Heterogeneity magnifies the tradeoffs between desiderata, so examining a particular system in detail might lead to successful explanations and predictions concerning that system, yet does not warrant explanations and predictions in other systems. Matthewson asks us to consider a group of aeroplanes such as the Airbus A380. They are complex but homogeneous entities, i.e. each Airbus is very similar to the next. Thus "it is possible to model their properties precisely, realistically and in a way that generalises across all of them.” (331). In contrast, a marine ecosystem and a forest ecosystem might have similar trophic levels, but the entities in each level are very different. Thus, the precise specification of the entities in one system will not generalize to the other.

I agree with Matthewson's insight concerning the importance of heterogeneity for tradeoffs, yet I think that there are three issues that merit some additional attention. First, Matthewson's conception of heterogeneity is ontological, as it refers to differences in the nature of systems' parts. Thus, airplane parts are the same type of thing across different systems, whereas the components of ecosystems (e.g. trees, fish, nitrogen, grasses, bacteria, invertebrates) are different types of things. 
The worry with focusing on ontological differences between systems is that they are not the only way in which heterogeneity can manifest. I will now turn to Aldo Leopold's notion of interdependence. ${ }^{3}$ For Leopold, interdependence is a set of positive and negative causal interactions between parts of an ecosystem, usually in different trophic levels (Leopold 1949/2014). He argued that an ecosystem should be understood as a system whose "functioning depends on the cooperation and competition of its diverse parts" (p. 116).

The important aspect of Leopold's conception, for this discussion, is the shift of focus away from the individual itself to its role in the structure of the community. Food chains are "lines of dependency" that "conduct energy upward", while death returns energy to the soil (p. 116). The ontology of the particular organisms within these food chains is much less important than the ways in which they absorb, transform and exude energy. The existence of various organisms and changes in the composition of these organisms have certain effects on the system, and these effects seem to be what Leopold thinks we should focus on. In other words, ecosystems are parcelled into causes.

If we parcel ecosystems into causes, we can examine the similarities and differences between systems in terms of how their parts are qua causes. Thus, rather than focusing on ontological heterogeneity, we focus instead on causal heterogeneity (Elliott-Graves 2016). This can be seen quite clearly in instances of trophic cascades, where predators have indirect effects on populations further down the trophic chain, by changing the abundance or

\footnotetext{
${ }^{3}$ I am indebted to Roberta Millstein for bringing the notion of interdependence to my attention.
} 
behaviour of their prey. For example, the abundance of St. John's wort is limited by the abundance of pollinator populations (mainly bees). Bees are subject to predation by dragonflies, so systems with fewer dragonflies have higher populations of bees and, consequently, St. John's wort. However, according to Knight et al. (2005), studies of these systems usually focus on the terrestrial part (plants \& their pollinators), which is problematic because examining the terrestrial systems does not reveal why there are differences in dragonfly abundances. Knight et al. found that it was the indirect effect of the presence or absence of fish in the adjacent ponds (that prey on the aquatic larval dragonflies, but not adults) that determined the abundance of plants in each system. In other words, parcelling the system into causes and identifying causal differences between parts of the system facilitated the provision of a full explanation of the phenomenon.

Of course, ontologically distinct parts often have different effects; therefore ontological heterogeneity can contribute to causal heterogeneity. In the previous example, the ontological differences across ponds (presence vs. absence of fish) precipitated the causal differences between the various systems. Nonetheless, some systems exhibit causal heterogeneity despite being ontologically similar. For instance, in plant-soil feedback (PSF) ${ }^{4}$ the same species of plants interact with the same species of soil microbes across different systems (e.g. geographically distinct prairies), yet these interactions are sometimes positive, sometimes

\footnotetext{
${ }^{4}$ Plants interact with microbes in the soil, e.g. arbuscular mycorrhizal fungi and nutrient fixing bacteria. These interactions can be beneficial or detrimental to the plant's growth. Often, different feedback loops cancel each other out resulting in apparent neutral feedback.
} 
neutral and sometimes negative (Klironomos 2002). Also, ontologically distinct systems sometimes exhibit causal homogeneity in some respects. For example, in cases of high niche overlap, different species fulfil very similar functions within the ecosystem (e.g. sardines vs. anchovies in marine trophic chains) (Ricklefs and Miller 2000). In short, ontological heterogeneity is neither necessary nor sufficient as a concept for capturing the differences between systems.

The second way to refine Matthewson's account is to distinguish between intra- and intersystem heterogeneity. Matthewson refers to inter-system heterogeneity, i.e. the heterogeneity between different systems such as different airliners or ecosystems. However, causal heterogeneity can also manifest within each system, as differences between the parts of a system can have different effects. For example, PSF can change within a particular system even when the composition of species remains constant. For example, exotic plant species often experience negative feedback with soil microbes when they first move to a new area, but then begin to experience positive feedback in the same system (van der Putten 2013).

This point is quite significant for explaining another difficulty in generalization, namely generalization into the future. Just as causal differentiation across systems makes it difficult to project knowledge from one system onto the next, causal differentiation within a system across time makes it difficult to project knowledge into the future -i.e. make predictions. Once again, this expectation is resonant in Leopold's notion of interdependence. He points out that changes in compositions of "floras and faunas" have effects that are "seldom intended or 
foreseen; they represent unpredicted and often untraceable readjustments in the structure" (Leopold 1949, 117).

A third issue in Matthewson's account concerns the particular desiderata that trade off against each other. For Matthewson, the salient tradeoff that gets magnified because of heterogeneity is the one between generality and precision. He points out that the properties of ontologically similar systems, such as airliners and electrons can be "modeled precisely" (331) and also generalized, whereas "ecosystems vary with respect to many of their important properties ... the more precise the model, the fewer ecosystems to which it applies" (331).

While I agree that ontological heterogeneity can result in this particular tradeoff, I believe that causal heterogeneity usually magnifies the tradeoff between generality and realism. As outlined above, a model's realism can be increased by including more of the real-world causal factors or by representing them with fewer distortions. Yet the process of generalizing often involves the opposite, i.e. omitting factors that are particular to each instance of a phenomenon so as to focus on what is common between the various instances (Cartwright 1994). This is, for instance, the rationale behind Type I models.

This explains why, in some cases, generalizations are possible. If the systems in question are causally homogeneous, then omitting details from the model is usually not problematic. That is, if the causal factors of the phenomenon are the same across systems, then we only need to identify them and include them in our models in order to have an accurate causal picture of the phenomenon. Technically, generalization is achieved at the expense of realism, 
because details are left out, yet this does not decrease the epistemic power of our model, precisely because they are details and leaving them out does not matter. For example, a computational model of Airbus380 flight must identify all the causal factors that affect flight (such as wind direction and speed, velocity, acceleration, overall weight), yet need not include details (such as logo of each plane, the gender composition of the passengers, the relative weights of passengers to cargo $)^{5}$. If the causal factors are identified correctly, then the model is applicable to all planes of that make, and all flights with environmental variables that fall within the parameters of the model. Of course, if relevant causal factors are omitted from homogeneous systems, then the model results will be less accurate. However, the point is that in causally homogeneous systems the differences between systems are details, not causal factors, thus, by focusing on what is common between systems we are much more likely to identify the relevant causal factors giving rise to the phenomenon.

The problem in cases of causal heterogeneity is that the differences between systems are much more often differences in terms of relevant causal factors, not mere details. The 'idiosyncrasies' of each system, i.e. the aspects of a system not shared by other systems, are not irrelevant details, but factors that affect the functioning of the system. In other words, idiosyncrasies in causally heterogeneous systems are not the equivalent of different colours of

\footnotetext{
${ }^{5}$ One could argue that some of these details can affect the airplane's flight (e.g. light refraction from different colours, slight differences in weight distribution etc.). Nonetheless, these effects, if present, are very small, and thus legitimately negligible. Any variation in flight time or fuel consumption because of differences in the colour of logos on airplane wings is overshadowed, or even cancelled out by the effects of relevant causal factors.
} 
the airplane logos, but the equivalent of different wing types or engines. Abstracting away these types of differences, by averaging them out or by omitting them altogether, can make a model's results highly and dangerously inaccurate.

A representative ecological example of causal heterogeneity and its effect on model accuracy, is drought sensitivity. Even though drought affects all plant communities to some extent, different plants employ radically different strategies for dealing with it, depending on factors such as climatic conditions (repeated short-term vs. long term water stress), plant demographic traits (age, height) and below-ground traits and interactions (root shape \& size, PSF interactions) (Phillips et al. 2016) . According to Phillips et al., the standard framework for examining drought-sensitivity suffers from "surface bias", focusing predominantly on hydraulic properties of leaves and stems and ignoring belowground interactions (310). This, they argue, has led to inaccuracies in model explanations and predictions, that have resulted in important gaps in our knowledge of drought sensitivity, despite its increasing importance.

Why does the standard framework omit belowground interactions? It is not because ecologists think that these factors are negligible details. Rather, below-ground interactions are so diverse that most models can only include highly abstracted versions of these interactions (Phillips et al. 2016). In other words, causal heterogeneity has forced these ecologists to choose between reducing the generality of their models (by including factors specific to particular systems) or their realism (by omitting the idiosyncratic factors). Most ecologists opt 
for reducing realism, which as Phillips et al. pointed out, is not always the best option. I will examine their alternative proposal in the next section.

\section{Common Interdependencies and Modest Generalisations}

Before discussing the alternative proposal, it might be useful to take a step back and review why scientists value generality in the first place. Generality is usually considered a desideratum because it allows scientists to compare disparate phenomena. Merely observing various phenomena, even in great detail, is somehow not seen as sufficiently scientific, because it does not show us how they connect to other phenomena -hence the denunciation of lawless ecology as mere 'stamp collecting'. Scientists also often worry that the absence of a general framework lessens the grounds for making or having confidence in one's predictions.

These ideas have also been popular in philosophy of science. Traditionally, the very process of identifying patterns across phenomena constituted a scientific explanation (Hempel and Oppenheim 1948; Kitcher 1981). The simpler the framework (in terms of the number of explanatory principles and 'brute facts' it appealed to) and the greater the number of phenomena it could explain, the more explanatorily powerful it was. A strong connection between grounds for predictability and explanatory power is also highlighted in some of these positions.

More recently, the popularity of these views has waned. They have been criticized from a number of standpoints (Salmon 1999; Cartwright 1983) and there has been an increased 
appreciation for the value of case studies and their importance for scientific research, especially in the study of complex and heterogeneous systems (Morgan 2015). Of course, this has not eclipsed the importance of generalizations completely; extremely localized results are still considered problematic (Guala 2003).

Nonetheless, this means that we can prise apart the notion of generality from the notions of unification, laws and symmetry with predictions, and assess the value of generality per se. This is important because the level of generality necessary for unification is much more extensive than the levels required to overcome extreme localism. A moderate level of generality, constrained to within particular types of phenomena or systems is often sufficient to provide the explanatory and predictive power ecologists expect of their models.

Returning to the example, Phillips et al. (2016) examined whether drought-sensitivity models could be improved by becoming more realistic (including belowground factors). They point out that some models are capable of distinguishing between up to 15 different plant functional types, each with their own parameterizations, though very few of these parameters are below the ground. They argue that this is easy to remedy, as the models can accommodate a large number of parameters, while the data for these parameters is relatively easy to acquire. In addition, they suggest choosing models that can integrate hydrology sub-models, as these can predict the availability of water for a plant given soil texture. Ultimately their aim is to identify combinations of plant and soil traits that confer sensitivity to drought across systems. 
The important points of the example for this discussion are the following. First, the way in which Phillips et al. advocate for the inclusion of belowground factors places a high importance on capturing the interdependencies between causal factors. The models they advocate for do not preclude that there is more than one combination that confers sensitivity to drought, rather they highlight the fact that different plants employ different strategies for different conditions. That is, these models do not include all the possible below ground factors and dynamics at once. This would constitute an increase in both realism and generality, which would be convenient, but unfortunately unfeasible for the reasons described in this paper.

Rather, they are flexible, in the sense that each time a model is applied to a particular system it includes certain below-ground factors or dynamics -those relevant for each case. This flexibility increases the realism of the models, by accurately capturing the relevant belowground causal factors.

Second, the increase in realism comes at the expense of extensive generality. The models do not identify a single trait or type of soil that causes the phenomenon in all cases. In fact, the generality of these models is explicitly constrained. They are only meant to apply to a subset of climatic conditions (drought) and to forests (rather than other ecosystems) -a far stretch from searching for a single theory to encompass all ecological interactions. The authors acknowledge the importance of striking "a balance between generality (e.g., by including representations of ecosystem processes based on first principles) and specificity (e.g., by 
capturing the unique biotic and abiotic attributes of a given site)" (317) and urge against going too far in either direction.

Consequently, a moderate level of generality is maintained. It comes in the form of bottom-up generalizations based on combinations of plant and soil traits that appear in more than one system. Scientists can gain valuable information from identifying these similarities, even if they are few and far between, especially if they are distant or ontologically diverse. For example, if a particular combination is useful for plants in systems with highly variable temperatures, this may provide information for how plants in a different system (with the same plant-soil trait combination) could deal with increasing temperature variability due to climate change. As long as the limited scope of these generalizations is recognized, i.e. their application is highly selective and carefully considered, these types of modest generalizations can be a fruitful tool for ecological research.

\section{Conclusion}

The quest for ecological generality has become more constrained, reflecting a greater appreciation of the complex and interdependent nature of ecological systems. This trend is also supported by a theoretical argument: ecological systems are often causally heterogeneous, so increasing generality results in decreasing the ability of models to include enough factors to provide accurate representations of the causal networks giving rise to a phenomenon. This reduces the models' explanatory and predictive power, as demonstrated by the example of 
drought sensitivity. Instead more modest generalizations do not result in oversimplification of causal networks and thus preserve the accuracy of a model's explanations and predictions, while also helping to overcome localism and any worries that ecology is anything like mere stamp collecting.

\section{Bibliography}

Beckage, Brian, Louis Gross, and Stuart Kauffman. 2011. "The Limits to Prediction in Ecological Systems.” Ecosphere 2 (11):125

Borer, Elizabeth, Stanley Harpole, Peter Adler, Eric Lind, John Orrock, Eric Seabloom, and Melinda Smith. 2014. "Finding Generality in Ecology: a Model for Globally Distributed Experiments." Methods in Ecology and Evolution 5 (1): 65-73.

Cartwright, Nancy. 1983. How the Laws of Physics Lie. Oxford University Press. 1994. Nature's Capacities and Their Measurement. OUP Catalogue

Casper, Brenda and Jeffrey Castelli. 2007. "Evaluating Plant-Soil Feedback Together with Competition in a Serpentine Grassland." Ecology Letters 10 (5): 394-400.

Colyvan, Mark and Lev Ginzburg. 2003. "Laws of Nature and Laws of Ecology." Oikos 101 (3): 649-53.

Cooper, Gregory. 1998. "Generalizations in Ecology: a Philosophical Taxonomy." Biology \& Philosophy 13 (4) 555-86.

Elliott-Graves, Alkistis. 2016. 'The problem of Prediction in Invasion Biology' Biology and Philosophy 31(3):373-393

Evans, Matthew, et al. 2013. "Do Simple Models Lead to Generality in Ecology?." Trends in Ecology \& Evolution 28 (10) 578-83.

Grimm, V, E Revilla, U Berger, F Jeltsch, W Mooij, S Railsback, H Thulke, J Weiner, T Wiegand, and D DeAngelis. 2005. "Pattern-Oriented Modeling of Agent-Based Complex Systems: Lessons From Ecology" Science 310 (5750). United States: 987-91.

Guala, Francesco 2003. Experimental Localism and External Validity Philosophy of Science 70(5) 1195-1205

Hempel, Carl, and Paul Oppenheim. 1948. "Studies in the Logic of Explanation." Philosophy of Science 15 (2). 135-75.

Kelly, J, and K G Horton. 2016. "Toward a Predictive Macrosystems Framework for Migration Ecology." Global Ecology and Biogeography.

Kingsland, S 1995. Modeling Nature. Chicago: University of Chicago Press.

Kitcher, Philip. 1981. "Explanatory Unification.” Philosophy of Science 48 (4): 507-31.

Klironomos, John 2002. "Feedback with Soil Biota Contributes to Plant Rarity and 
Invasiveness in Communities." Nature 417 (6884) 67-70.

Knight, Tiffany, Michael McCoy, Jonathan Chase, Krista McCoy and Robert Holt. 2005.

'Trophic cascades across ecosytsems' Nature 437(7060): 880-883

Lawton, John 1999. "Are There General Laws in Ecology?.” Oikos 84 (2): 177-92.

Leopold, Aldo. 1949. “The Land Ethic.” In The Ecological Design and Planning Reader

(2014), edited by Forster O Ndubisi, 108-21. Island Press/Center for Resource

Economics.

Levins, Richard 1966. "The Strategy of Model Building in Population Biology." American

Scientist 54 (4): 421-31.

R. 1993. "A Response to Orzack and Sober: Formal Analysis and the Fluidity of Science" Quarterly Review of Biology. 68(4) 547-555

Linquist, Stefan, Ryan Gregory, Tyler Elliott, Brent Saylor, Stefan Kremer, and Karl Cottenie. 2016. "Yes! There Are Resilient Generalizations (or 'Laws') in Ecology." The Quarterly Review of Biology 91 (2) 119-31.

Matthewson, John. 2011. "Trade-Offs in Model-Building: a More Target-Oriented Approach." Studies in History and Philosophy of Science Part A 42 (2): 324-33.

May, Robert. 2004. "Uses and Abuses of Mathematics in Biology" Science 303 (5659).

American Association for the Advancement of Science: 790-93.

Mikkelson, Gregory M. 2001. "Complexity and Verisimilitude: Realism for Ecology." Biology \& Philosophy 16(4) 533-46.

Mitchell, Sandra. 1997. "Pragmatic Laws.” Philosophy of Science. 64:468-479

Morgan, Mary 2015. "Case Studies: One Observation or Many? Justification or Discovery?" Philosophy of Science 79(5)667-677

Odenbaugh, Jay. 2003. "Complex Systems, Trade-Offs, and Theoretical Population Biology:

Richard Levin's "Strategy of Model Building in Population Biology" Revisited."

Philosophy of Science (Proceedings) 70 (5): 1496-1507.

Peters, D, B Bestelmeyer, and J Herrick. 2006. "Disentangling Complex Landscapes: New

Insights Into Arid and Semiarid System Dynamics." BioScience 56(6) 491-501

Peters, R. 1991. A Critique for Ecology. Cambridge: Cambridge University Press.

Phillips, R, I Ibanez, and L D'Orangeville. 2016. "A Belowground Perspective on the Drought

Sensitivity of Forests: Towards Improved Understanding and Simulation." Forest Ecology and Management 380, 309-320

Railsback, Steven, and Volker Grimm. 2011. Agent-Based and Individual-Based Modeling: a Practical Introduction. Princeton University Press.

Richardson, David, and Marcel Rejmánek. 2004. "Conifers as Invasive Aliens: a Global

Survey and Predictive Framework." Diversity and Distributions 10 (5-6) 321-31.

Ricklefs, Robert, and Gary Miller. 1999 Ecology. New York: Freeman

Salmon, Merrilee H. 1999. Introduction to the Philosophy of Science. Hackett Publishing. Shrader-Frechette, K, and E McCoy. 1993. Method in Ecology. Cambridge University Press. Thrush, S, J Hewitt, V Cummings, and M Green. 2000. "The Generality of Field Experiments: 
Interactions Between Local and Broad-Scale Processes.” Ecology 81(2) 399-415.

Travis, J, F Coleman, and P Auster. 2014. "Integrating the Invisible Fabric of Nature Into Fisheries Management (Proceedings of the National Academy of Sciences of the United States of America (2014) 111, 2

Valéry, Loïc, Hervé Fritz, and Jean-Claude Lefeuvre. 2013. "Another Call for the End of Invasion Biology." Oikos 122 (8): 1143-46.

Warren, Jeffrey, Paul Hanson, Colleen Iversen, Jitendra Kumar, Anthony Walker, and Stan Wullschleger (2015) "Root Structural and Functional Dynamics in Terrestrial Biosphere Models - Evaluation and Recommendations." New Phytologist 205 (1): 59-78.

van der Putten, et al. (2013). Plant-soil feedbacks: the past, the present and future challenges. Journal of Ecology, 101(2), 265-276. 